\title{
Eine neue volumetrische Methode von allgemeiner Anwendbarkeit.
}

\author{
Von \\ Launcelot W. Andrews. ${ }^{1}$
}

Wenn Kaliumjodid in neutraler Lösung mit Chlorwasser titriert wird, bis das als Indikator zugesetzte Chloroform entfärbt ist, so findet bekanntlich eine der Gleichung

$$
\mathrm{KJ}+3 \mathrm{Cl}_{2}+3 \mathrm{H}_{2} \mathrm{O}=\mathrm{KCl}+\mathrm{HJO}_{3}+5 \mathrm{HCl}
$$

entsprechende Reaktion statt.

Andererseits ist es vielleicht nicht so wohl bekannt, dafs bei Gegenwart eines grofsen Überschusses von Salzsäure bei der Titration, unter Anwendung von Chloroform oder Kohlenstofftetrachlorid als Indikator, die Reaktion im Sinne der folgenden Gleichung verläuft:

$$
\mathrm{KJ}+\mathrm{Cl}_{2}=\mathrm{KCl}+\mathrm{JCl}
$$

In beiden Fällen wird die Beendigung der Reaktion durch die Entfärbung der nicht mit der Lösung mischbaren Flüssigkeit gekennzeichnet.

Die nach Gleichung 1 verlaufende Reaktion ist bereits vor langer Zeit von A. und F. DupkE ${ }^{2}$ zur Titration von Jodiden verwendet worden.

Versuch 1. Um den Einfluis von Salzsäure auf den Verlauf der Reaktion festzustellen, titrierte ich $5 \mathrm{ccm}$ einer $1 / 10$-norm. Kaliumjodidlösung in Gegenwart von $5 \mathrm{ccm}$ Chloroform mit Chlorwasser. Nach Zusatz von $75.4 \mathrm{ccm}$ Chlorwasser wurde das Chloroform farblos. Die Titration wurde jetzt mit weiteren $5 \mathrm{ccm}$ der

1 Ins Deutsche übertragen nach dem Manuskript von J. Koppes.

${ }^{2}$ Lieb. Ann. 94 (1855), 365. 
Jodkaliumlösung unter Zusatz von 15, 20 und $30 \mathrm{ccm}$ starker Chlorwasserstoffsäure ausgeführt; es wurden verbraucht $25.04,25.22$ und $25.25 \mathrm{ccm}$ Chlorwasser. Das Ende der Reaktion war sehr scharf zu erkennen.

Demnach ist zur Vollendung der Reaktion bei Abwesenheit von Chlorwasserstoffsäure ungefähr dreimal so viel Chlorwasser erforderlich als bei Gegenwart überschüssiger Salzsäure, wie es auch die Theorie verlangt. Es würde wahrscheinlich die theoretische Menge Chlorwasser $(75.75 \mathrm{ccm})$ verbraucht werden, wenn die durch die Reaktion selbst erzeugte relativ geringe Menge Chlorwasserstoffsäure (Gleichung 1) durch Zusatz von Calciumkarbonat neutralisiert würde.

Versuch 2. Um den Einflufs kleinerer Säuremengen festzustellen, wurde die Titration wie vorher wiederholt, nur wurden je 2,5 und $10 \mathrm{ccm}$ konzentrierte Chlorwasserstoffsäure zugesetzt; verbraucht wurden $34.1,26.9,26.0$ und $25.6 \mathrm{ccm}$ Chlorwasser.

Arbeitet man anstatt mit Chlorwasser mit einer Lösung von Kaliumjodat, so hängt die Stufe, auf der die Reaktion stehen bleibt, gleichfalls von der Konzentration der Säure ab. Ist diese gering, so wird nur das Jod freigemacht, entsprechend der folgenden Gleichung:

$$
5 \mathrm{KJ}+\mathrm{KJO}_{3}+6 \mathrm{HCl}=6 \mathrm{KCl}+3 \mathrm{~J}_{2}+3 \mathrm{H}_{2} \mathrm{O} ;
$$

ist jedoch ein grofser Salzsäureüberschufs vorhanden, so verläuft die Reaktion:

$$
2 \mathrm{KJ}+\mathrm{KJO}_{3}+6 \mathrm{HCl}=3 \mathrm{KCl}+3 \mathrm{JCl}+3 \mathrm{H}_{2} \mathrm{O}
$$

Im ersteren Falle (Gleichung 3) bleibt das nicht mit dem Wasser mischbare Lösungsmittel violett, im letzteren wird es farblos, während die darüberstehende Lösung sich durch das Chlorjod hellgelb färbt. - Die wahrscheinliche Erklärung dieses Verhaltens ist, dals das Jodchlorid als Salz einer sehr schwachen Base in neutraler oder schwach saurer Lösung der Hydrolyse unterliegt, wobei ein Hydroxyd und eine Säure entsteht, und zwar nach der Gleichung

$$
\mathrm{JCl}+\mathrm{H}_{2} \mathrm{O}=\mathrm{JOH}+\mathrm{HCl}
$$

das Jodhydroxyd (die unterjodige Säure), welches einer spontanen Umwandlung in Jodsäure u. s. w. anheimfällt. Durch einen grofsen Überschufs von Salzsäure dagegen wird die Hydrolyse zurück- 
gedrängt. Die Richtigkeit dieser Anschauung wird sowohl durch die bereits beschriebenen als auch durch die folgenden Versuche bestätigt. - Die Vorteile, die sich für praktische $Z$ wecke aus dem Ersatz des Chlorwassers durch Kaliumjodatlösung ergeben, sind ohne weiteres klar, und die bereits erwähnten Versuche gaben Anlafs zu der Hoffnung, dafs die Verhinderung der Hydrolyse des Jodchlorids sich praktisch vollständig durchführen liefse. Die folgenden Versuche zeigen, dafs dieses erreicht werden kann.

\section{Bestimmung von Jodiden.}

Versuch 3. Eine $1 / 5$-norm. Jodatlösung mit 9.7465 g reinem sauren Kaliumjodat $\left[\mathrm{KH}\left(\mathrm{JO}_{3}\right)_{2}\right]$ im Liter wurde hergestellt; nach der Theorie (Gleichung 4) entspricht jeder ccm dies Lösung $16.60 \mathrm{mg}$ Kaliumjodid oder $12.685 \mathrm{mg} \mathrm{J}_{\mathrm{od}}$ in einem Jodid. $10 \mathrm{ccm}$ einer Kaliumjodidlösung, welche pro Liter $20.6000 \mathrm{~g}$ reines Salz enthielt, wurden in eine mit Glasstopfen versehene Flasche von etwa $250 \mathrm{ccm}$ Inhalt zusammen mit etwa $5 \mathrm{ccm}$ Chloroform, $20 \mathrm{ccm}$ Wasser und $30 \mathrm{ccm}$ Chlorwasserstoffsäure (spez. Gew. 1.21) gebracht und das Gemisch mit der $1 / 5$-norm. Jodatlösung titriert, bis das Chloroform entfärbt war. Nach jedem Jodatzusatz wurde heftig geschüttelt; der Endpunkt der Reaktion liefs sich sehr scharf erkennen. Erforderlich waren $12.43 \mathrm{ccm}$ der Jodatlösung, also wurden $157.7 \mathrm{mg}$ oder $76.54 \%$ Jod gefunden. Nach der Theorie sind im Jodkalium $76.42 \%$ Jod.

$\nabla$ ersuch 4. Angewandt wurden $15 \mathrm{ccm}$ Jodlösung und $33 \mathrm{ccm}$ Chlorwasserstoffsäure ohne Zusatz von Wasser; im übrigen wurde die Titration ganz wie bei Versuch 3 ausgeführt. Verbraucht wurden $18.62 \mathrm{ccm}$ Jodatlösung, entsprechend $236.2 \mathrm{mg}$ oder $76.42 \%$ Jod.

Versuch 5. Angewandt wurden $25 \mathrm{ccm}$ der Jodidlösung, $50 \mathrm{ccm}$ Chlorwasserstoffsäure, kein Wasser; im übrigen wurde wie bei 3 gearbeitet. Verbraucht wurden $31.15 \mathrm{ccm}$ der Jodatlösung, entsprechend $395.1 \mathrm{mg}$ oder $76.72 \%$ Jod.

Versuch 6. Es wurden $30 \mathrm{ccm}$ der Jodidlösung und $50 \mathrm{ccm}$ Chlorwasserstoffsäure ohne weiteren Wasserzusatz wie bei Versuch 3 titriert. Es waren erforderlich $37.12 \mathrm{ccm}$ der Jodatlösung, die $470.0 \mathrm{mg}$ oder $76.19 \%$ Jod entsprachen.

Aus diesen Versuchen ergaben sich die folgenden praktischen Vorschriften. Die Lösung des zu bestimmenden Jodids ist mit einer 
genügenden Menge konzentrierter Chlorwasserstoffsäure anzusäuern, so dals am Ende der Titration in der Lösung wenigstens $15 \% \mathrm{HCl}$ enthalten sind; eine grölsere Menge schadet nichts. Nach Zusatz von $5 \mathrm{ccm}$ Chloroform wird in einer mit Glasstopfen verschlossenen Flasche mit $1 / 10^{-}$oder $1 / 5$-norm. Jodatlösung titriert, bis das Chloroform entfärbt ist.

\section{Bestimmung von freiem Jod.}

Es ist klar, dafs freies Jod nur halb so viel einer solchen Jodatlösung verbraucht wie die gleiche Quantität Jod in Form von Jodid; daher ist ein ccm der $1 / 5$-norm. Jodatlösung $25.370 \mathrm{mg}$ freiem Jod äquivalent. Das Jod kann in Form einer Lösung in Schwefelkohlenstoff, Chloroform, Tetrachlorkohlenstoff", Benzol u. s. w. oder in fester Form vorhanden sein. Die Titration wird ebenso wie bei der Bestimmung der Jodide ausgeführt. $\mathrm{Zu}$ der folgenden Titration wurde eine Lösung von bekanntem Gehalt an Jodid und Jod verwendet.

Versuch 7. Angewandt wurden $5 \mathrm{ccm}$ der Jodidlösung, $344.7 \mathrm{mg}$ Jod, $10 \mathrm{ccm}$ Chlorwasserstoffsäure und $5 \mathrm{ccm}$ Chloroform; im übrigen wurde wie bei 3 gearbeitet; $19.85 \mathrm{ccm}$ Jodat waren erforderlich. Da von diesem $6.20 \mathrm{ccm}$ für das Jodid verbraucht wurden, so kommen auf das freie Jod $13.65 \mathrm{ccm}$ entsprechend $346.3 \mathrm{mg}$. Dies Resultat ist etwas zu hoch, zweifellos weil nicht genügend Salzsäure angewendet worden war. Es sollten wenigstens $20 \mathrm{ccm}$ zugesetzt werden.

\section{Bestimmung von Chromaten.}

Das Chromat wird in einer Jodkaliumlösung von bekanntem Gehalt aufgelöst und zwar verwendet man wenigstens etwas mehr als drei Moleküle Jodid auf je zwei Moleküle Chromsäure. Nach Zusatz von $5 \mathrm{ccm}$ Chloroform und soviel rauchender Salzsäure, dafs diese nach der Titration dem halben Volumen der Flüssigkeit entspricht, titriert man wie gewöhnlich mit Jodat auf farblos. Fehlende Salzsäure kann noch während der Titration ergänzt werden. Die Differenz zwischen dem für das Jodid allein erforderliche Jodat und der für Jodid und Chromat gemeinsam verbrauchten Menge ist dem letzteren äquivalent.

Versuch 8. Angewandt wurden $5 \mathrm{ccm}$ Kaliumjodidlösung (vgl. Versuch 3) $36.8 \mathrm{mg}$ Kaliumdichromat, $5 \mathrm{ccm}$ Wasser, $20 \mathrm{ccm}$ 
Chlorwasserstof'säure. Verbraucht wurden $2.53 \mathrm{ccm}$ Jodatlösung. Da ron der letzteren $6.20 \mathrm{ccm}$ dem Jodid entsprechen, so entfallen $3.67 \mathrm{ccm}$ auf das Chromat; diese zeigen $36.3 \mathrm{mg}$ Kaliumdichromat an.

\section{Bestimmung von Chloraten.}

Die Titration wird in derselben Weise wie bei den Chromaten ausgeführt. Nach dem Ansäuern mit der vorgeschriebenen Salzsäuremenge lälst man das Gemisch in dicht geschlossener Flasche etwa 10 Minuten stehen, damit die Reaktion vollständig zu Encie geht. Ein weiteres vierundzwanzigstündiges Stehen ändert die Resultate nicht. Für jedes Molekül Chlorsäure müssen mehr als 3 besser noch 4 - Moleküle Jodid verwendet werden.

Versuch 9. Es wurden angewandt: 5 ccm einer Kaliumchloratlösung mit $70.3 \mathrm{mg}$ Salz, $25 \mathrm{ccm}$ Jodidlösung (vgl. Versuch 3), $50 \mathrm{ccm}$ Chlorwasserstoffsäure und $5 \mathrm{ccm}$ Chloroform. Nachdem das Gemisch 15 Minuten im verschlossenen Gefüfs gestanden hatte, wurde mit $1 / 5$-norm. Jodatlösung titriert. Verbraucht wurden $13.65 \mathrm{ccm}$. Da für das Jodid allein $31.00 \mathrm{ccm}$ erforderlich waren, so entfallen auf das Chlorat $17.35 \mathrm{ccm}$, entsprechend $70.90 \mathrm{mg}$.

Die Versuche 10 und 11 waren dem letzten ähnlich; nur wurde die Dauer der Reaktion auf 1 Minute abgekürzt bezw. sofort titriert. Fs wurden nur 69.80 bezw. $69.55 \mathrm{mg}$ Chlorat gefunden.

Versuch 12. Ein Gemisch von $5 \mathrm{ccm}$ der Chloratlösung, $25 \mathrm{ccm}$ der Jodidlösung und $40 \mathrm{ccm}$ Salzsäure blieb 24 Stunden stehen und erforderte sodann $13.77 \mathrm{ccm}$ Jodat, welche $70.41 \mathrm{mg}$ Kaliumchlorat entsprachen.

Versuch 13. Zur Titration eines Gemisches ron $5 \mathrm{ccm}$ einer Kaliumchloratlösung (33.46 mg Chlorat), $10 \mathrm{ccm}$ der Jodidlösung und $20 \mathrm{ccm}$ Chlorwasserstoff'säure, welches 10 Minuten stehen blieb, wurden verbraucht $4.23 \mathrm{ccm}$ Jodatlösung. Da für das Jodid allein $12.40 \mathrm{ccm}$ erforderlich waren, so entfielen auf das Chlorat $8.17 \mathrm{ccm}$, was $33.39 \mathrm{mg} \mathrm{KClO}_{3}$ entspricht.

\section{Bestimmung von Antimon und Arsen.}

Es war sehr wahrscheinlich, dafs in Gegenwart von genügenden Mengen Chlorwasserstoffsäure zwischen Jodsäure und Antimontrioxyd oder Arsentrioxyd der folgenden Reaktion quantitativ verläuft 


$$
\begin{aligned}
&-81- \\
& 2 \mathrm{AsCl}_{3}+\mathrm{KJO}_{3}+5 \mathrm{H}_{2} \mathrm{O}=2 \mathrm{H}_{3} \mathrm{AsO}_{4}+\mathrm{KCl}+\mathrm{JCl}+4 \mathrm{HCl} \\
& 2 \mathrm{SbCl}_{3}+\mathrm{KJO}_{3}+5 \mathrm{H}_{2} \mathrm{O}=2 \mathrm{H}_{3} \mathrm{SbO}_{4}+\mathrm{KCl}+\mathrm{JCl}+4 \mathrm{HCl} .
\end{aligned}
$$

Vorläufige orientierende Versuche zeigten nun, dafs bei Gegenwart von Arsen oder Antimon, wenn zuviel Salzsäure vorhanden war, die Endreaktion undeutlich wird, was wahrscheinlich auf Dissoziationserscheinungen der Pentachloride dieser Elemente zurückzuführen ist. Es zeigte sich, dafs $30 \%$ Chlorwasserstoffsäure - berechnet auf das Gewicht der ganzen Flüssigkeitsmenge am Schlusse der Titration - über die zulässige Maximalgrenze hinausgehen, während dies für $25 \%$ nicht der Fall ist; die Minimalgrenze liegt andererseits nahe bei 12 oder $15 \%$ Säure. Man siebt also, dafs die geeignete Konzentration der Chlorwasserstoffsäure auf ziemlich enge Grenzen beschränkt ist, die allerdings nicht so nahe bei einander liegen, dals beim Arbeiten tatsächlich Schwierigkeiten entstehen. Die Schärfe der Endreaktion wird bei keinem bekannten volumetriscben Verfahren übertroffen, wenn Bromide oder sonstige färbende in Chloroform lösliche Substanzen ausgeschlossen werden.

Versuch 14. Angewandt wurden $20 \mathrm{ccm}$ Wasser, $30 \mathrm{ccm}$ Chlorwasserstoffsäure und $25 \mathrm{ccm}$ Antimonlösung, die im Liter $31.251 \mathrm{~g}$ sorgfältig; gereinigtes Kalium-Antimonyltartrat enthielt $(0.7813 \mathrm{~g}$ Salz in $25 \mathrm{ccm})$. Verbraucht wurden $23.62 \mathrm{ccm}$ Jodatlösung, die $283.9 \mathrm{mg}$ Antimon entsprechen. Es wurden also $36.34 \%$ Antimou gefunden, währond $36.13 \%$ berechnet sind.

Versuch 15. Es wurden angewandt $25 \mathrm{ccm}$ der erwähnten Antimonyllösung und $25 \mathrm{ccm}$ Chlorwasserstoffsäure. Zur Titration waren $23.50 \mathrm{ccm}$ Jodat erforderlich, die $282.5 \mathrm{mg}$ Antimon entsprechen; gefunden also $36.16 \% \mathrm{Sb}$.

Versuch 16. Angewandt wurden $25 \mathrm{ccm}$ Arsentrioxydlösung und $50 \mathrm{ccm}$ Chlorwasserstoffsäure; verbraucht wurden $24.4 \overline{\mathrm{ccm}}$ Jodatlösung, die $242.1 \mathrm{mg}$ Arsentrioxyd entsprechen. Durch Titration mit Jodlösung in Gegenwart von Bikarbonat in der üblichen Weise ergab sich, dafs dio Natriumarsenitlösung $243.8 \mathrm{mg}$ Arsentrioxyd enthielt.

Versuch 17. Zur Titration eines Gemisches von $5 \mathrm{ccm}$ der Arsenitlösung, $10 \mathrm{ccm}$ Wasser und $5 \mathrm{ccm}$ Chlorwasserstoffsäure waren erforderlich $4.91 \mathrm{ccm}$ Jodatlösung, die $48.6 \mathrm{mg} \mathrm{As} \mathrm{O}_{3}$ äquivalent sind. Jodometrisch wurden gefunden $48.8 \mathrm{mg}$.

z. anorg. Chom. Bd. 36. 
Versuch 18. Angewandt: $20 \mathrm{ccm}$ Arsenitlösung, $40 \mathrm{ccm}$ Chlorwasserstoffsäure. Verbraucht $19.69 \mathrm{ccm}$ Jodat, entsprechend $194.9 \mathrm{mg}$ Arsentrioxyd. Jodometrisch fanden sich $194.7 \mathrm{mg}$.

Versuch 19. Angewandt: $15 \mathrm{ccm}$ Arsenitlösung und $30 \mathrm{ccm}$ Chlorwasserstoffsäure. Zur Titration verbraucht $14.79 \mathrm{ccm}$ Jodat, entsprechend $146.4 \mathrm{mg}$ Arsentrioxyd. Die jodometrische Titration ergab $146.3 \mathrm{mg}$.

Bei diesen Versuchen blieb das Gemisch 10 bis 60 Minuten vor der Titration stehen, ausgenommen bei Nr. 16, wo bereits nach etwa einer Minute titriert wurde. 15 Minuten sollen jedoch mindestens bis zur Titration vergehen. Kupfer stört die Titration von Arsen oder Antimon nicht. Das Arsen in Pariser Grün und ähnlichen Verbindungen kann sehr schnell und genau bestimmt werden, indem man die gewogene Substanz in $50 \mathrm{ccm}$ Salzsäure (1.1 spez. Gew.) in einer verschlossenen Flasche löst, $5 \mathrm{ccm}$ Chloroform und sodann bis fast zur Entfärbung des letzteren aus einer Bürette Jodatlösung hinzufügt. Nachdem das Gemisch 15 Minuten gestanden hat, wird die Titration in der gewöhnlichen Weise zu Finde geführt.

Versuch 20. $20 \mathrm{ccm}$ der Arsenitlösung wurden wie bei Versuch 18 titriert und erforderten $8.95 \mathrm{ccm}$ Jodat. Das gleiche Volumen dieser Lösung. wurde mit $1 \mathrm{~g}$ Kupfersulfat versetzt und erforderte sodann $9.00 \mathrm{ccm}$ Jodat zur Titration. In beiden Fällen waren $20 \mathrm{ccm}$ rauchende Chlorwasserstoffsäure vorhanden.

\section{Bestimmung von Eisen.}

Jodchlorid wirkt auf Ferrosalze oxydierend und diese Reaktion kann zur Titration der letzteren in derselben Weise benutzt werden, wie für die Bestimmung des Antimons bereits beschrieben. Die Endreaktion ist langsam und entbehrt der Schärfe, welche die anderen Titrationen auszeichnet. Wenn die Färbung des Chloroforms durch Zusatz von Jodat zerstört ist, so kehrt sie allmählich zurück. Zusatz von Manganochlorid scheint für die Verschärfung der Endreaktion von Vorteil zu sein, doch bedarf dieser Punkt noch der näheren Aufklärung. Gegenwart von Oxalsäure beeinflufst die Resultate nicht, wie die folgenden Beleganalysen zeigen.

Versuch 21. Angewandt wurden $0.2492 \mathrm{~g}$ Ammoniumferrosulfat, $15 \mathrm{ccm}$ der Jodlösung, $30 \mathrm{ccm}$ Salzsäure und ein kleiner Krystall von Manganosulfat. Verbraucht wurden $21.77 \mathrm{ccm}$ Jodatlösung, von denen 18.60 für das Jodid erforderlich waren, so dafs 
$3.17 \mathrm{ccm}$ für das Eisen verblieben. Dies entspricht $35.44 \mathrm{mg}$ Eisen oder $14.22 \%$. Theorie: $14.25 \%$.

Versuch 22. Angewandt wurden $2.0874 \mathrm{~g}$ Ammoniumferrosulfat, $20 \mathrm{ccm}$ Wasser und $30 \mathrm{ccm}$ Chlorwasserstoffsäure. Beim Titrieren mit einer Kaliumjodatlösung (B) die $2.10 \%$ stärker war als eine $1 / 5$-norm. Lösung, von der also $1 \mathrm{ccm} 11.42 \mathrm{mg}$ Eisen äquivalent war, wurden $26.06 \mathrm{ccm}$ verbraucht, entsprechend $297.6 \mathrm{mg}$ Eisen oder $14.26^{\circ} \%$.

Versuch 23. Es wurden $2.0843 \mathrm{~g}$ Ammoniumferrosulfat und ca. $1 \mathrm{~g}$ Oxalsäure, das übrige wie bei Nr. 22 angewandt. Verbraucht wurden $25.95 \mathrm{ccm}$ der Jodatlösung B, welche $296.3 \mathrm{mg}$ oder $14.22 \%$ Eisen entsprechen.

Die hier beschriebene Methode ist ebenso allgemeiner Anwendung fähig wie die Bunsensche Destillationsmethode; die Resultate sind von derselben Genauigkeit wie die der letzteren; aufserdem kann man sie einfacher und schneller erhalten. In verschiedenen Fällen, wo Bunsens Verfahren nicht anwendbar ist, so z. B. bei der Titration von Arsen oder Antimon in Gegenwart von Kupfer läfst sich die hier beschriebene Methode ohne weiteres ausführen.

Chemical Laboratory of the University of Jowa, 5. Mai 1903.

Bei der Redaktion eingegangen am 19. Mai 1903. 